\title{
Germanica
}

\section{De Vienne à Berlin, de l'essai sur Hofmannsthal à $L a$ dégradation des valeurs (H. Broch) - en passant par Paris (M. Proust, J. Dos Passos)}

Von Wien nach Berlin, vom Essay über Hofmannsthal bis zum Zerfall der

Werte (H. Broch) - über Paris (M. Proust, J. Dos Passos)

\section{Vincent Ferré}

\section{(2) OpenEdition}

\section{Journals}

Édition électronique

URL : http://journals.openedition.org/germanica/548

DOI : $10.4000 /$ germanica. 548

ISSN : 2107-0784

Éditeur

Université de Lille

Édition imprimée

Date de publication : 1 décembre 2008

Pagination : 29-39

ISBN : $978-2-913857-22-6$

ISSN : 0984-2632

Référence électronique

Vincent Ferré, « De Vienne à Berlin, de l'essai sur Hofmannsthal à La dégradation des valeurs (H. Broch) - en passant par Paris (M. Proust, J. Dos Passos) », Germanica [En ligne], 43 | 2008, mis en ligne le 01 décembre 2010, consulté le 06 octobre 2020. URL : http://journals.openedition.org/germanica/548 ; DOI : https://doi.org/10.4000/germanica.548

Ce document a été généré automatiquement le 6 octobre 2020.

(c) Tous droits réservés 


\section{De Vienne à Berlin, de l'essai sur Hofmannsthal à La dégradation des valeurs (H. Broch) - en passant par Paris (M. Proust, J. Dos Passos)}

Von Wien nach Berlin, vom Essay über Hofmannsthal bis zum Zerfall der

Werte (H. Broch) - über Paris (M. Proust, J. Dos Passos)

\section{Vincent Ferré}

Deux textes de Hermann Broch, Les Somnambules (Die Schlafwandler : Eine Romantrilogie, 1931-1932) et l'essai Hofmannsthal et son temps (Hofmannsthal und seine Zeit, 1947-1950), permettent d'envisager le couple formé par les deux villes «à part " ${ }^{1}$ que sont Berlin et Vienne, moins en termes de présence que de disparition, ou du moins d'abstraction dans leur représentation. La comparaison sera double, à la fois générique - confrontation d'un essai et d'un roman du même auteur - et culturelle, par le rapprochement entre Broch et des écrivains d'une autre nationalité (Proust, pour À la recherche du temps perdu etDos Passos, pour USA) écrivant à propos d'une autre capitale (Paris): il s'agira d'envisager le lien entre la part d'abstraction dans la représentation de la ville et l'intégration d'une forme de théorie au sein du roman, qui constitue une caractéristique marquante de ces trois œuvres publiées au cours de la même période (le premier tiers du $\mathrm{xx}^{\mathrm{e}}$ siècle). Selon l'hypothèse que je souhaite vérifier, cette abstraction - qu'on définira, pour le moment, de manière minimale comme une stylisation, une représentation épurée ${ }^{2}$ - est due à la présence, au sein des œuvres, des passages théoriques (la «Dégradation des Valeurs" dans le cas des Somnambules) qui ressemblent à des essais et échangent des traits avec ce roman qui les accueille. 


\section{Berlin, ville abstraite?}

2 La trilogie des Somnambules se déroule en partie à Berlin, dans Pasenow et dans Huguenau. C'est dans cette ville que s'ouvre l'incipit - à travers la déambulation de M. von Pasenow ${ }^{3}$-, relayé par une série de noms évoquant des rues et des lieux publics biens connus : Friedrichstrasse, Wintergarten, Unter den Linden, chez Dressel, l'opéra ; on trouve aussi des références plus vagues aux faubourgs ${ }^{4}$ ou aux demeures des personnages. Dans Huguenau, «L'histoire de la jeune Salutiste de Berlin », qui inscrit de manière significative la ville dans son titre même, évoque le foyer de l'héroïne, l'immeuble où demeure Bertrand Müller, des cafés, etc.; lui répondent en écho des séquences de la « Dégradation des Valeurs » (toujours dans Huguenau), qui font allusion aux tramways dans les rues, aux grands magasins. On comprend que certains lecteurs aient donc pu être sensibles aux accents réalistes de la narration encadrante, par la description qui est faite de l'Empire sous Guillaume II, de la fin du XIX ${ }^{e}$ siècle à 1918 - à l'instar des critiques contemporains, qui ont parfois comparé Broch à Fontane ou Heinrich Mann.

3 Toutefois, aller trop loin dans cette lecture revient à ignorer les limites de la représentation : que voit-on de Berlin, dans la troisième partie des Somnambules? Les lieux sont présentés selon une perspective relative et ce, dès le début de Pasenow,ce trait ne faisant que s'accentuer par la suite. Dans cette première partie des Somnambules, les épisodes se déroulant à la Bourse ou à l'Opéra sont avant tout révélateurs des obsessions du protagoniste: au Faust qu'il voit représenté se mêlent ainsi des hallucinations transformant deux spectateurs en images de Ruzena, sa future maitresse, ce qui joue sur sa mauvaise conscience ( $"$ Cet effroi était bien gratuit et plus gratuit encore lui parut le sentiment d'infidélité à l'égard d'Elisabeth qui lui succéda », 38/39-40).

4 On parlera ici de relativité en mobilisant la célèbre analyse proposée par l'essai sur "James Joyce et le temps présent ", qui établit une double comparaison, rapprochant la physique et le roman classiques d'une part, d'autre part la théorie de la relativité (la seule à tenir compte de l'observateur) et le roman moderne, qui met en question l'évidence du langage et de la représentation. Les premiers se limitaient (selon Broch) à " placer l'objet purement et simplement dans le cône de l'observation et [...] le décrire purement et simplement $»^{5}$; au contraire, dans le roman moderne, "le sujet d'observation, donc le "narrateur en tant qu'idée" [der "Erzähler als Idee"], tout autant que le langage avec lequel il décrit l'objet représenté y ont leur place comme intermédiaires de la représentation $»^{6}$, ce qui induit un biais. Ce biais éclaire alors le caractère somnambulique de Pasenow dans la première partie ou encore l'insistance sur la problématique religieuse dans Huguenau, qui confèrent au décor un caractère irréel et stylisé, épuré, encore plus marqués. L'abstraction apparaît en effet de plus en plus prononcée au fil des volumes : seuls quelques traits sont conservés, isolés, séparés du reste - abstraits, si l'on songe au sens étymologique de séparation, d'isolement, par opposition avec ce qui apparaît comme concret, proche du monde sensible.

Il serait possible de montrer qu'une telle impression de lecture est confortée par les prises de position de Broch, qui ne s'inscrit dans une esthétique "réaliste » qu'en donnant un sens très large à ce terme et en l'opposant à la tentative de reproduction pure et simple du réel. Mais on s'en tiendra ici à cette première justification du terme d' abstraction, dont la valeur heuristique est confirmée par son utilisation ponctuelle chez 
des critiques frappés par ce « décor métropolitain anonyme, [cette] capitale moderne quelconque et représentative en soi $»^{7}$. Ainsi de Dieter Hornig, qui souligne les limites du réalisme supposé des Somnambules dans la description de Berlin, la mettant - comme d'autres commentateurs, mais sans justifier cette association - en relation avec la Vienne de l'essai sur Hofmannsthal : " ces deux villes forment un cadre fantomatique sans accéder à une épaisseur réaliste. L'œuvre de Broch possède une topographie particulièrement floue. Elle est seulement traversée d'effets de réel, qui forment un faible contrepoids au caractère abstrait de ces textes $»^{8}$. Hornig en reste toutefois à cette remarque - qui paraphrase Broch, comme on va le voir -, son dessein étant avant tout de montrer la présence cryptée de Vienne derrière la ville (Berlin) évoquée explicitement dans Les Somnambules.

6 Or l'on peut plutôt s'interroger sur la raison de cette abstraction, sur ce choix esthétique de Broch; d'autant qu'elle est centrale dans Les Somnambules, et ne concerne pas le seul décor. Elle va en effet de pair avec une abstraction du narrateur et des personnages, et elle est renforcée par une dimension onirique. Pour n'évoquer ici que le premier point (on reviendra plus loin sur l'onirisme), on rappellera que les interventions du narrateur ont été supprimées au fil de la genèse des Somnambules, pour aboutir à sa quasi-disparition dans les deux premières parties. En outre, bien que le narrateur prenne dans Huguenau l'apparence de Bertrand Müller', celui-ci est très peu décrit, selon le souhait explicite de Broch : s'il remanie la version de 1930 pour éclairer davantage cette figure, comme l'attestent les épreuves, sa correspondance témoigne de sa volonté de ne " pas [l]' “incarner" davantage » dans Pasenow, par le biais d'un portrait ou par son langage. Bertrand - dans son avatar nommé von Bertrand conservera finalement « une légère tournure à l'abstraction [mit einer kleinen Wendung zum Abstrakten] $\aleph^{10}$.

7 Ce terme, que je reprends ici pour lire Les Somnambules, Broch l'utilise de manière répétée dans un de ses textes les plus célèbres, qu'il convient de prendre en compte pour examiner l'évocation de la ville, cette fois dans son écriture essayistique.

\section{Vienne, capitale du vide}

8 Comme souvent, comprendre un texte de Broch demande de passer par un autre de ses écrits : le rapprochement avec Hofmannsthal et son temps (Hofmannsthal und seine Zeit) peut permettre de mieux cerner cette abstraction. La manière dont Vienne est convoquée dans cet essai (en particulier dans ses deux premiers chapitres ${ }^{11}$ ), les modalités de la référence à cette ville dans un texte non-fictionnel, peuvent-elles expliquer les particularités de la représentation de la ville dans le roman des Somnambules, dans ses chapitres diégétiques comme dans la Dégradation des valeurs, texte théorique inclus dans Huguenau et qui rappelle le genre de l'essai ${ }^{12}$ ?

9 Hofmannsthal n'est mentionné qu'au bout d'une cinquantaine de pages (p. 100), avant que ne commence la partie proprement biographique (p. 108 sq.) ${ }^{13}$; c'est donc Vienne qui a la prééminence. Les longues analyses du «non style » du XIX ${ }^{e}$ siècle, de l'art pour l'art, de la cruauté de l'époque, du rapport de l'art au mythe et à l'art primitif, la font apparaître d'abord en filigrane, avant que l'évocation de "l'apocalypse joyeuse » (die fröhliche Apokalypse, 79/145, traduction modifiée) ne la fasse passer au premier plan. Mais la ville est réduite à quelques traits, négatifs et exprimés par les mêmes termes, ressassés: la première description insiste sur l'impression produite par Vienne, 
d'emblée connotée négativement ("atmosphère sinistre", 51/115) et des termes récurrents disent le vide, décliné dans les domaines politique, artistique (par l'absence d'art authentique) : «En sa qualité de capitale du kitsch, Vienne devint également celle du vide de valeurs de son époque » (108/175, traduction modifiée).

Or, de manière frappante pour nous, le terme employé comme un leitmotiv pour dire ce vide est précisément l'adjectif abstrait [abstrakt]. Abstraction de la politique de François Joseph d'une part : «politique d'orientation abstraite », mené par celui qui a un " goût [...] pour l'abstraction » (89/156); « Plus François Joseph Ier vieillit, plus il plongea dans le vide de sa mission [...] et [...] devait supporter sa part d'isolement abstrait » (97/163). De la révolution de 1848 ensuite : « la révolution viennoise était aussi abstraite que le régime qu'elle combattait " (92/159). Abstraction, enfin et plus généralement, de la situation politique, qui est marquée par un déséquilibre entre les nationalités et que Broch considère en elle-même comme une «création abstraite " $(95 / 161)$ : «Ce qui subsistait encore de l'État autrichien c'était le fantôme, le squelette d'une théorie à laquelle personne ne croyait plus. / L'État était une chose et les activités politiques dont il était le théâtre en étaient une autre. Entre les deux, il y avait une espèce de couche isolante impénétrable qui, manifestement, rendait abstrait chacun d'eux" (95-96 /162). La dizaine d'occurrences, en moins de vingt pages, indique le caractère répétitif du texte, ressassement de formules et d'idées proches, avec des variations : ce martèlement, une constante de l'écriture de Broch puisque on la retrouve aussi bien dans ses essais que dans ses textes diégétiques, dessine l'image d'une ville vide, stylisée. Or ce même type de représentation traverse les parties diégétiques des Somnambules, on l'a vu, et La dégradation des valeurs, séquences théoriques incluses dans le roman, comme on va le constater.

11 Si l'on visualise les trois pôles: l'essai autonome (sur Hofmannsthal), la diégèse du roman encadrant, et la Dégradation des valeurs qui se situe à l'intersection, on peut avancer l'hypothèse que l'intégration ${ }^{14} \mathrm{~d}^{\prime} u n$ essai sur la dégradation des valeurs a contaminé la diégèse : apportant avec eux une manière propre à l'essai de représenter la ville, ces chapitres théoriques ont provoqué un échange de certains traits avec ce roman qui les accueille, ce qui peut expliquer le caractère abstrait commun aux chapitres, qu'ils soient diégétiques ou théoriques. (Pourquoi parler d'échange? Parce que l'essai inclus dans le roman a, réciproquement, été modifié par cette fictionnalisation, devenant narratif et fictionnel ${ }^{15}$ ).

12 Pour faire l'essai de cette hypothèse, et préciser les contours de Vienne comme de Berlin dans les parties théoriques et diégétiques des Somnambules, un détour géographique et littéraire par Paris va permettre de mieux cerner le caractère abstrait du décor, qui forme le pendant de celles des personnages et du narrateur.

\section{De Berlin à Vienne en passant par Paris (Proust et Dos Passos)}

13 À la recherche du temps perdu de Marcel Proust (publiée entre 1913 et 1927) et USA de John Dos Passos (trilogie publiée entre 1930 et 1936, reprise en un volume en 1938) présentent des similitudes frappantes avec Les Somnambules, de par l'abstraction du narrateur, le caractère fantomatique des personnages et de certains lieux. Ainsi, l'omniprésence du narrateur proustien ne le rend-elle pas difficile à percevoir, paradoxalement? Sarraute a sans doute été l'une des premières à souligner la 
contrepartie de cette visibilité (il occupe tout l'espace du texte), en évoquant dans L'Ère $d u$ soupçon les œuvres dans lesquelles un «être sans contours, indéfinissable, insaisissable et invisible, un "je" anonyme [...] a usurpé le rôle du héros principal »" Évoquantl'« "abstraction" du héros ", son "absence de caractérisation et [son] retrait par rapport au monde ", Florence Godeau lui répond lorsqu'elle transpose le titre de Musil au cas du narrateur proustien et remarque que le moi, dans la Recherche, est " "sans qualités" »".

14 Cette particularité du narrateur trouve son pendant chez les personnages, qui ne sont que des fantômes privés d'existence propre, des "ombres", des "visions, rêves, cauchemars, illusions » (Sarraute) ${ }^{18}$. Le terme de «fantômes » est d'ailleurs employé par le narrateur pour désigner les personnes qu'il "[poursuit]», telles $\mathrm{M}^{\mathrm{me}} \mathrm{de}$ Guermantes ou Albertine, «des êtres dont la réalité pour une bonne part était dans [s]on imagination $»^{19}$; et comme en écho, le narrateur de l'«Histoire de la Jeune Salutiste " compare Marie et Nussim à des fantômes [Spuk] (552/551). Mais, plus globalement, l'on songe, chez Broch, à la manière dont le passage au second plan de von Bertrand, dans la deuxième partie, tout comme sa "rencontre » onirique avec Esch, accentuent de façon comparable le caractère évanescent du personnage, qui finit par disparaître du roman à sa mort. Dans le volume médian, le Président von Bertrand, inaccessible à Esch même au siège de la Mittelrheinische Reederei, ressemble au dieu invisible $^{20}$ évoqué par la Dégradation des valeurs, dans laquelle la question de l'abstraction revient d'ailleurs comme un leitmotiv ${ }^{21}$. C'est également, pour finir, un point commun avec U.S.A., qui a marqué ses commentateurs à cet égard. En 1932, John Chamberlain estime que les personnages sont utilisés comme des «symboles » mais n'ont aucune profondeur ${ }^{22}$. Cette remarque revient avec insistance dans la critique, chez Sartre comme chez Claude-Edmonde Magny, du côté français. Pour cette dernière, les personnages de U.S.A. ne se caractérisent que par des états physiques, leur langage étant fait de clichés : «ils ne sont littéralement rien, que des paquets d'opinions toutes faites, d'idées reçues d'un groupe social ou d'un autre, en même temps qu'un vocabulaire $»^{23}$. Comme la figure imaginée par le narrateur proustien, les personnages de Dos Passos n'auraient ainsi pas de «caractère $»^{24}$. Tel est le sentiment partagé par Hemingway, à en croire une lettre où il reproche aux personnages de 1919 d'avoirtrop peu de réalité, de réalité physique, d'être presque « symboliques » ${ }^{25}$.

15 Enfin, si la ville offre un cadre spatial commun aux trois récits, cette ville apparaît d'une façon fragmentaire par les aperçus qu'en ont les personnages qui déambulent ou la contemplent. Paris est bien «le cœur du monde » et le cœur du récit chez Proust et Dos Passos; mais ne sont gardés d'elle que quelques traits, souvent altérés. Sa représentation révèle d'une part une distorsion temporelle analogue : à la comparaison qu'établit Le Temps retrouvé entre 1916 et leDirectoire répond, dans 1919 un rapprochement entre la capitale et l'Antiquité - qui sert précisément de référence à la mode du Directoire. D'autre part, critiques et lecteurs désignent du nom d'irréalité ou d' onirisme ce qui suscite une impression d'étrangeté et s'apparente manifestement à de l'abstraction.

On se souvient de la comparaison proposée par Sartre à propos de USA : «N'y aurait-il pas eu de tour de passe-passe? Je regarde autour de moi : des gens, des villes, des bateaux, la guerre. Mais ce ne sont pas les vrais: ils sont discrètement louches et sinistres, comme dans les cauchemars $\aleph^{26}$. Cette image précise le sentiment de critiques perplexes devant l'«atmosphère très particulière » de U.S.A., où la présence de 
l'onirisme est plus homogène que dans Manhattan Transfer ${ }^{27}$. Lorsqu'il s'est agi de transposer à l'écran la Recherche, où le décor prend par moments un aspect irréel comme lors des alertes aériennes dans le Paris nocturne du Temps retrouvé, comparé au Bagdad des Mille et une Nuits ou à un opéra de Wagner ${ }^{28}-$, c'est ce caractère onirique et irréel que Volker Schlöndorff a retenu, pour son adaptation d'Un Amour de Swann. Il s'agissait pour lui de mettre à l'écran « un Paris inhabituel, presque fantasmagorique " ${ }^{29}$. Dans ses « Notes de travail », le cinéaste précise ainsi son impression d'un flottement dans la géographie et la chronologie du roman proustien : «On croit toujours s'éveiller en sortant d'une lecture de Proust. Ce texte sans anecdote est situé au cœur du monde intérieur où temps et espace deviennent relatifs au point de se confondre. Peut-être le film devrait-il être un songe ${ }^{30}$. Même si Un Amour de Swann lui apparaît comme particulier dans la Recherche, en ce que des lieux précis sont évoqués (quai d'Orléans, Opéra, Saint-Germain, entre autres), Schlöndorff estime que le Paris de Proust ne fait pas penser «à une ville réelle " comme celui de Balzac ou de Zola, mais à un lieu "imaginaire ", irréel, évoquant pour lui Marx Ernst et Chirico ${ }^{31}$. Il ne s'agit pas de souscrire totalement à ce jugement, mais de rendre compte de l'impression ressentie par un lecteur qui l'a exprimée à son tour dans son adaptation cinématographique.

Une impression de lecture initiale, qui percevait le caractère abstrait de la diégèse et en particulier (pour s'en tenir à l'objet du colloque) de la représentation de la ville de Berlin dans les Somnambules, s'est trouvée corroborée non seulement par les déclarations de Broch et des remarques convergentes de critiques, mais aussi par une autre impression, suscitée par les romans de Proust et de Dos Passos, comparables par leur inscription historique et la place qu'ils accordent à la théorie. D'où l'hypothèse proposée ici, selon laquelle ce caractère abstrait a à voir avec la présence, dans le roman, de parties théoriques. La prise en compte de l'écriture essayistique de Broch, dans son étude sur Hofmannsthal, fait apparaître des points communs dans la représentation de la ville, Vienne ou Berlin, dans l'essai ou dans le roman, comme si l'écriture essayistique, présente dans la fiction par l'intermédiaire de la Dégradation des valeurs, avait coloré, contaminé l'ensemble des Somnambules. Ou plutôt, comme si les deux pôles, théorique et diégétique, avaient échangé des qualités : l'essai intégré dans le récit se transformant, la diégèse devenant abstraite, épurée, y compris dans sa représentation de l'espace.

18 Faute de place, je me suis limité au seul essai sur Hofmannsthal, mais il aurait été possible de montrer la permanence de cet intérêt de Broch pour l'abstraction, qui apparaît encore dans ses réflexions sur le "style de vieillesse », cette rupture dans l'expression, cette "brutale rupture de style ", qui peut être " décrite comme une sorte d'abstraction où l'expression repose de moins en moins sur le vocabulaire [...] et [...] de plus en plus sur la syntaxe $\aleph^{32}$. Parmi les exemples convoqués, deux références picturales importantes mériteraient notre attention : d'une part les « peintres français du début du siècle", qui "furent les premiers à prendre conscience que tout le vocabulaire naturaliste, inévitablement, était devenu périmé, et qu'il fallait trouver l'essentiel même au prix de devenir abstrait "; d'autre part Picasso, qui a su dans Guernica saisir l'essence de l'époque ${ }^{33}$.

La notion d'abstraction paraît enfin d'autant plus utile qu'elle sert souvent à désigner les textes théoriques, publiés sous forme d'essais autonomes ou insérés dans un roman ; dans le cas présent, elle met en évidence le continuum existant entre diégèse et théorie, dans Les Somnambules. Le roman de Broch n'est pas isolé, dans la littérature de 
l'époque, et passer par Paris a mis en perspective cette œuvre avec des expérimentations romanesques contemporaines. Il serait possible à présent d'envisager l'existence de particularités des romans de langue allemande, en considérant d'autres textes et d'autres types d'espaces : plus qu'à Berlin Alexanderplatz de Döblin (1929) - où Franz Biberkopf, sortant de prison, retrouve un monde extérieur qui lui paraît étrange et menaçant ${ }^{34}$ - qui ne contient pas de théorie comparable, on songe bien sûr à Musil et la scène d'ouverture de l'Homme sans qualités, ainsi qu'à La Montagne magique de Thomas Mann (1924), où la « Digression sur le temps » est liée à la contemplation de l'espace: "C'est au fond une aventure singulière que cette acclimatation à un lieu étranger... $»^{35}$.

\section{NOTES}

1. Voir l'introduction aux actes du colloque Wien-Berlin, Deux sites de la modernité - Zwei Metropolen der Moderne (1900-1930) organisé à Montpellier en 1992 par M. Godé, I. Haag et J. Le Rider (Cahiers d'études germaniques, 1993, n²4,p. 5).

2. Il aurait été possible de contextualiser davantage la référence à l'abstraction, du côté du domaine pictural et des analyses que Broch en propose (voir par exemple la note en conclusion); mais cette intervention a recherché un équilibre entre la contextualisation (historique et culturelle, du côté germanophone) et une approche générique et comparatiste.

3. «En 1888, M. von Pasenow avait soixante-dix ans. Certaines gens éprouvaient un inexplicable sentiment d'antipathie, dans les rues de Berlin, à son approche [...]» (Les Somnambules [1956-1957], éd. revue et augmentée, trad. de Pierre Flachat et Albert Kohn, Paris, Gallimard, coll. «L'Imaginaire», 1990, p.11; Die Schlafwandler: Eine Romantrilogie [1931-1932], éd. de P. M. Lützeler, Francfort, Suhrkamp, 1976 (1986), p. 11). La première référence paginale renvoie à la version française, la seconde au texte original des Somnambules (abrégés en $S$ lorsque la référence n'est pas évidente).

4. Respectivement p. 18-19, 29-30 (18-19, 30-31).

5. H. Broch, "James Joyce et le temps présent », in Création littéraire et connaissance [désormais abrégé en CLC], éd. et introduction de H. Arendt, trad. d'A. Kohn, Paris, Gallimard, 1966 (1985), p. 201 (" James Joyce und die Gegenwart », in Schriften zur Literatur. I. Kritik, éd. de P. M. Lützeler, Francfort, Suhrkamp, 1975 (1986), p. 78).

6. Ibid.

7. D. Hornig, «Hermann Broch et Les Somnambules : Berlin, scène imaginaire », in Wien-Berlin, op. cit., p. 101.

8. Ibid.

9. Je simplifie ici volontairement un problème largement traité dans la critique brochienne (voir les références canoniques: Th. Ziolkowski, «Zur Entstehung und Struktur von Hermann Brochs ,Schlafwandlern'», Deutsche Vierteljahresschrift für Literaturwissenschaft und Geistesgeschichte, 1964, XXXVIII, p. 57 et "Hermann Broch and Relativity in Fiction », Wisconsin Studies in Contemporary Literature, 8, 1967, p. 206-216 ; K. R. Mandelkow, Hermann Brochs Romantrilogie Die Schlafwandler, Gestaltung und Reflexion im modernen deutschen Roman, Heidelberg, Carl Winter, 1962, p. 150-152), sans reprendre l'argumentation que j'ai proposée dans « De Charybde en Scylla : une réduction 
de la fracture polytextuelle est-elle possible ? (Dos Passos, Broch)», à lire en ligne sur la page $<$ http://www.univ-paris13.fr/cenel/articles.htm>.

10. Lettre au Rhein-Verlag du 24 juin 1930 (Lettres (1929-1951), éd. de Robert Pick, trad. d'A. Kohn, Paris, Gallimard, 1961, p. 25 (Briefe 1 (1913-1938), Dokumente und Kommentare zu Leben und Werk, éd. de P. M. Lützeler, Francfort, Suhrkamp, 1981 (1986), p. 92).

11. Rappelons le caractère composite de l'essai sur Hofmannsthal et son temps, formé (dans les éditions qui ont fait connaître Broch) à partir de deux textes : deux chapitres écrits en 1947-1948 et une introduction (1950) à une anthologie de textes en prose de Hofmannsthal, commandée à Broch par la fondation Bolligen. Le cœur de l'essai est bien constitué du premier bloc de deux chapitres, qui sont évoqués ici.

12. Ce sont les questions non génétiques, mais génériques, qui importent ici: que Les Somnambules soient antérieurs de 15 ans à l'essai sur Hofmannsthal n'est pas un problème, puisqu'il s'agit de confronter écriture romanesque et écriture essayistique. Hofmannsthal et son temps est choisi comme exemple représentatif de l'écriture non-fictionnelle de Broch, et parce qu'il est parfois rapproché des Somnambules par les critiques.

13. "Hofmannsthal et son temps ", CLC, p. 45-184 (« Hofmannsthal und seine Zeit. Eine Studie ", in Schriften zur Literatur. I. Kritik, op. cit., p. 111-334).

14. Sur le geste de Broch qui a intégré cet essai, et sur la genèse, voir par exemple M. Durzak

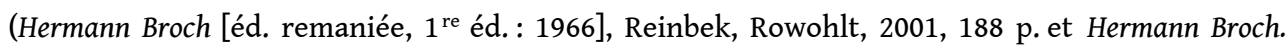
Dichtung und Erkenntnis, Stuttgart, Berlin, Cologne, W. Kohlhammer, 1978, p. 33-60).

15. Cette interprétation semble d'autant plus défendable que des observations comparables peuvent être faites sur USA et la Recherche, qui contiennent eux aussi des parties théoriques, fortement narratives: je ne reprends pas ici une démonstration proposée ailleurs (V. Ferré, L'Essai fictionnel chez M. Proust, H. Broch et J. Dos Passos, thèse de littérature générale et comparée, université Rennes 2, 2003, chapitre 6).

16. N. Sarraute, L'Ere du soupçon. Essais sur le roman [1956], Paris, Gallimard, coll. « Folio Essais ", 1987, p. 61.

17. F. Godeau, A la recherche du temps perdu de M. Proust et Der Mann ohne Eigenschaften de R. Musil : les désarrois du moi, thèse de littérature générale et comparée, université Paris 4, 1992, p. 427 et p. 12 - la première formule n'a pas été reprise dans la version publiée (Les désarrois du moi : «À la recherche du temps perdu » de M. Proust et «Der Mann ohne Eigenschaften » de R. Musil, Tübingen, M. Niemeyer, 1995, vii, 300 p.), la seconde se trouve p. 5.

18. N. Sarraute, op. cit., p. 61-62.

19. M. Proust, Sodome et Gomorrhe, in A la recherche du temps perdu, édition publiée sous la direction de J.-Y. Tadié, Paris, Gallimard, coll. « Bibliothèque de la Pléiade », 1988, vol. 3, p. 401.

20. Et ce à plus d'un titre, puisque la présence de traits divins a été mise en lumière chez ce personnage (voir D. Cohn, The Sleepwalkers, Elucidations of Hermann Broch's Trilogy, La Hague-Paris, Mouton \& Co., 1966, p. 76).

21. Voir les chapitres 34 ( «la raison première fut transportée [...] vers le véritable infini abstrait », p. 471/474), 44 (sur la dissolution dans l'abstraction de l'image du monde), 62 (sur l'abstraction du protestantisme), etc.

22. J. Chamberlain, « News Novel », New York Times Book Review, 13 mars 1932, republié dans Dos Passos, The Critical Heritage, éd. de B. Maine, Londres-New York, Routledge, 1988, p. 105.

23. Cl.-E. Magny, L'Âge du roman américain, Paris, Seuil, coll. «Pierres vives », 1948 (1968), p. 132. Gide, de son côté, parle d' « êtres pulvérulents », dont « [a]ucun [...] ne [l']'intéresse » (Journal, II (1926-1950), éd. deM. Sagaert, Paris, Gallimard, coll. « Bibliothèque de la Pléiade », 1997, p. 449).

24. Cl.-E. Magny, L’Âge du roman américain, op. cit., p. 134.

25. Le terme est de Townsend Ludington, biographe de Dos Passos (The Fourteenth Chronicle. Letters and Diaries of John Dos Passos, éd. de T. Ludington, Boston, Gambit, 1973, p. 381). 
26. J.-P. Sartre, "À propos de John Dos Passos et de "1919" " [1938], in Situations I, Paris, Gallimard, 1947 (1992), p. 15.

27. P. Bleu-Schwenninger, John Dos Passos, L'écriture miroir, Grenoble, Ellug, 1993, p. 105. Elle évoque en particulier les séquences de «L'Æil-caméra » relatives aux images parentales.

28. Le Temps retrouvé, in À la recherche du temps perdu, op. cit., vol. 4,p. 388 et 338.

29. "Débusquer les gens et les choses. Entretien avec Jean-Claude Carrière ", L'Avant-scène cinéma, 321-322, 1984, p. 17.

30. Volker Schlöndorff, "À propos de l'adaptation d'Un amour de Swann. Notes de travail ", Bulletin de la société des amis de Marcel Proust et des amis de Combray, 34, 1984, p. 180.

31. Ibid., p. 182 (le terme onirique revenait fréquemment dans les discussions de Schlöndorff avec son chef opérateur). Ces remarques pourraient être rapprochées de l'importance accordée au rêve dans Les Somnambules et la Recherche ainsi qu'à l'analogie partielle entre rêve et littérature proposée par les deux romanciers.

32. "Le style de l'âge mythique ", CLC, p. 260 ("The Style of the Mythical Age ", introduction à Rachel Bespaloff, On The Iliad, 1947).

33. Ibid., p. 271.

34. «[...] les façades des maisons se succédaient sans arrêt. Et il y avait des toits sur ces maisons, des toits qui flottaient au-dessus d'elles. Ses yeux erraient jusque là-haut: "Pourvu que ces toits ne dégringolent pas!" Mais les maisons restaient bien droites.» (Alfred Döblin, Berlin Alexanderplatz, Paris, Gallimard, coll. « Folio », 1970 (1994), p. 22-23).

35. Thomas Mann, La Montagne magique, Paris, LGF, coll. « Livre de poche », 1997, p. 120.

\section{RÉSUMÉS}

La représentation de Vienne et de Berlin est ici envisagée dans une perspective de double confrontation, culturelle et générique. Sont comparés un essai et un roman de Hermann Broch (Hofmannsthal et son temps, Les Somnambules), entre eux puis avec les œuvres de deux écrivains contemporains dont les textes accordent une place importante à une autre capitale, Paris Proust et Dos Passos - afin d'examiner l'abstraction qui caractérise la représentation de la ville dans ces trois romans, associée à une atmosphère onirique ou irréelle. Le rapprochement avec l'essai de Broch vise à interroger la relation entre cette abstraction et la présence de passages théoriques au sein du roman (La dégradation des valeurs, dans Les Somnambules) - comme s'ils échangeaient des traits avec ce roman qui les accueille.

Die Darstellung der beiden Hauptstädte, Wien und Berlin, wird in diesem Beitrag unter dem Blickwinkel eines sowohl kulturellen als auch gattungszentrierten Vergleichs vorgenommen. Verglichen werden hier ein Essay und ein Roman von H. Broch (Hofmannsthal und seine Zeit, Die Schlafwandler) zuerst miteinander und dann mit Werken von zwei zeitgenössischen Autoren, deren Inhalt einer anderen europäischen Hauptstadt einen bedeutenden Platz einräumen und zwar Paris - Proust und Dos Passos -, um die Abstraktheit bei der Darstellung der Stadt mit einer unwirklichen und irrealen Stimmung in den drei Romanen näher in Augenschein zu nehmen. Der Vergleich mit H. Brochs Essay zielt auf ein kritisches Hinterfragen dieser Abstrahierung und ihres Zusammenhangs mit bestimmten theoretischen Passagen im Roman (Zerfall der Werte im Roman Die Schlafwandler). 
INDEX

Mots-clés : Vienne, Berlin

\section{AUTEURS \\ VINCENT FERRÉ}

Université de Paris 13 (CENEL) 